\title{
SPECTRAL ANALYSIS OF FINITE CONVOLUTION OPERATORS $\left({ }^{1}\right)$
}

\author{
BY \\ RICHARD FRANKFURT
}

\begin{abstract}
In this paper the similarity problem for operators of the form (*) $T: f(x) \rightarrow \int_{0}^{x} k(x-t) f(t) d t$ on $L^{2}(0,1)$ is studied. Let $K(z)=$ $\int_{0}^{1} k(t) e^{i t z} d t$. A function $C(z)$ is called a symbol for $T$ if $C(z)$ can be written in the form $C(z)=K(z)+e^{i z} G(z)$, where $G(z)$ is a function bounded and analytic in a half plane $y>\delta$, for some real number $\delta$. Under suitable restrictions, it is shown that two operators of the form (*) will be similar if they possess symbols which are asymptotically close together as $z \longrightarrow \infty$ in some half plane $y>\delta$.
\end{abstract}

In this paper we study operators on $L^{2}(0,1)$ of the form

$$
T: f(x) \rightarrow \int_{0}^{x} k(x-t) f(t) d t,
$$

where $k \in L^{1}(0,1)$. We are concerned with the question of when two such operators are similar; i.e., given two operators $T_{1}$ and $T_{2}$ of the form (1), under what circumstances will there exist an invertible operator $X$ on $L^{2}(0,1)$ such that $X T_{1}=T_{2} X$ ?

Earlier research in this area has been accomplished almost entirely by methods from the theory of integral equations, and dates back to the classical investigations of Volterra [28] and Volterra and Pérès [29]. While these techniques have the theoretical advantage of being equally applicable to Volterra operators with more general kernels (i.e., not necessarily depending only on the difference of the arguments), they also have the practical disadvantage of becoming extremely unwieldy in even the simplest cases. See Daniel [4], [5], Freeman [10], Kalisch [15], [16], [17], Kalmuševskii [18], Sahnovič [21], [22], [23], and for related problems Osher [20]. See also Gohberg and Kreìn [13].

In the particular case of operators of the form (1), an alternative approach is provided by the complex Fourier transform. However, while the technique itself is very old, its use in the systematic spectral analysis of operators of the form (1) seems to be something of a novelty. The initial step in this direction

Received by the editors October 7, 1974.

AMS (MOS) subject classifications (1970). Primary 47G05; Secondary 47B35, 30A36, 30A78.

(1) This paper constitutes a portion of the author's Doctoral Dissertation, written at the University of Virginia under the supervision of Professor James Rovnyak. 
was taken by Sarason in his short note [24], but without further development. In [9] a general technique for studying operators of the form (1), based upon the use of the complex Fourier transform, was constructed. The study of operators of the form (1) can in this way be subsumed in the study of compressions of analytic Toeplitz operators to $H^{2} \ominus B H^{2}$, where $B(z)$ is a singular inner function determined by a single point mass. In particular, this observation leads one to approach the problem of similarity via the mechanism of composition operators. We describe briefly the basic type of result that occurs.

If $T$ is any operator of the form (1), by a symbol for $T$ we mean any function $C(z)$ having a representation of the form $C(z)=K(z)+e^{i z} G(z)$, where $K(z)=\int_{0}^{1} k(t) e^{i t z} d t$, and $G(z)$ is bounded and analytic in some half plane $y>\eta$, $\eta$ a real number. It often happens that if $T_{1}$ and $T_{2}$ are operators of the form (1), $T_{1}$ and $T_{2}$ will be similar if they possess symbols $C_{1}(z)$ and $C_{2}(z)$, respectively, such that $C_{1}(z) / C_{2}(z) \rightarrow 1$ sufficiently rapidly as $z \rightarrow \infty$ in some half plane $y>\eta$. It is generally necessary to assume that one of the symbols has a particularly wholesome form. In [9] a result of this type was obtained for the case in which one of the symbols has the form $C(z)=L(-i z) /(-i z)^{\alpha}, \alpha>0$, where $L(z)$ is a function analytic and nonvanishing in a sector $|\arg (z-\eta)|<$ $\beta \pi / 2, \eta>0$ and $\beta>1$, with $L(x)>0$ for $x>\eta$ and $z L^{\prime}(z) / L(z) \rightarrow 0$ as $z \rightarrow \infty$ in the sector. Such a function was called slowly varying at infinity in the sector. This result was shown to contain as a special case the earlier theorem of Kalisch [15] and Sahnovič [22]. (Choose $L(z) \equiv 1$.) However, while perceptibly broader in scope than this earlier result, it is not nearly the most inclusive theorem that can be obtained.

In this present paper we prove a much more general result. The basic idea will be to develop a classification scheme for the asymptotic behavior of functions analytic in a half plane. This technique will then be used to identify pairs of similar operators of the form (1) by comparing the asymptotic behavior of their symbols. Numerous concrete examples will be provided to illustrate the applicability of the theory.

Some additional references on Volterra operators are the books of Brodskii [3] and Sz.-Nagy and Foiaş [27].

1. Preliminary results. For $0<p \leqslant \infty$, let $H^{p}$ denote the Hardy space of functions $F(z)$ analytic in the half plane $y>0$ such that

$$
\|F\|_{p}=\left\{\begin{array}{l}
\sup _{y>0}\left(\int_{-\infty}^{\infty}|F(x+i y)|^{p} d x\right)^{1 / p}, 0<p<\infty, \\
\sup _{y>0}|F(x+i y)|, \quad p=\infty,
\end{array}\right.
$$

is finite. By making the usual identification with boundary functions, we may 
alternately regard $H^{p}$ as a subspace of $L^{p}(-\infty, \infty)$. We use these notions interchangeably.

If $B(z)$ is any inner function, it is easily shown that $H^{2} / B H^{2}$ is a Hilbert space, and $H^{\infty} / B H^{\infty}$ is a commutative Banach algebra. $H^{\infty} / B H^{\infty}$ may further be identified with an algebra of operators on $H^{2} / B H^{2}$; namely, for any $A+B H^{\infty} \in$ $H^{\infty} / B H^{\infty}$ let $T\left(A+B H^{\infty}\right)$ be defined on $H^{2} / B H^{2}$ by

$$
T\left(A+B H^{\infty}\right): F+B H^{2} \rightarrow A F+B H^{2} .
$$

For general properties of these operators, the reader is referred to Sarason [25] and Frankfurt and Rovnyak [9].

We shall be concerned exclusively with the special case $B(z)=e^{i z}$. While elements of $H^{\infty} / e^{i z} H^{\infty}$ are not functions, it is nevertheless true that any two functional representatives of the same coset have similar asymptotic behavior as $y \rightarrow \infty$. From this it is reasonable to expect that the essential properties of a given coset should not be affected by the behavior of a specific representative in a region $y<\eta, \eta$ a real number. This idea can be made more precise. Specifically, in [9] the following notation was introduced.

Let $\Pi^{\infty}$ denote the set of all functions $F(z)$ bounded and analytic in a half plane $y>\eta$, where $\eta$ is a real number depending on $F$. We identify two such functions if one is an analytic continuation of the other. If $F$ and $G$ are bounded and analytic in half planes $y>\eta$ and $y>\delta$, respectively, and $a$ and $b$ are complex numbers, we define $a F+b G$ and $F G$ pointwise in the half plane $y>\max \{\eta, \delta\}$. With these conventions, $\Pi^{\infty}$ becomes a complex commutative algebra.

The following result holds:

LEMMA 1. The mapping $F+e^{i z} H^{\infty} \rightarrow F+e^{i z} \Pi^{\infty}$ is an algebra isomorphism of $H^{\infty} / e^{i z} H^{\infty}$ onto $\Pi^{\infty} / e^{i z} \Pi^{\infty}$.

Similarly, let $\Pi^{2}$ denote the set of all functions $F(z)$ for which $F(z+i \eta)$ $\in H^{2}$, for some real number $\eta$ depending on $F$. Again, we identify two such functions if one is an analytic continuation of the other. It is easy to see that $\Pi^{2} \subset \Pi^{\infty}$ (see Duren [6, p. 191]). We let $\Pi^{2}$ inherit the linear structure of $\Pi^{\infty}$. Thus $\Pi^{2}$ becomes a complex linear space.

LEMMA 2. The mapping $F+e^{i z} H^{2} \rightarrow F+e^{i z} \Pi^{2}$ is a linear isomorphism of $H^{2} / e^{i z} H^{2}$ onto $\Pi^{2} / e^{i z} \Pi^{2}$.

Lemmas 1 and 2 are proved in [9]. By using the isomorphisms of these lemmas, we may transfer the topological and metric structures of $H^{\infty} / e^{i z} H^{\infty}$ and $H^{2} / e^{i z} H^{2}$ to $\Pi^{\infty} / e^{i z} \Pi^{\infty}$ and $\Pi^{2} / e^{i z} \Pi^{2}$, respectively. These spaces may therefore be used in place of the spaces $H^{\infty} / e^{i z} H^{\infty}$ and $H^{2} / e^{i z} H^{2}$ in all that fol- 
lows. The advantage in so doing is that the notation is freed from dependence upon a particular half plane.

By Lemma 2, we observe that for any $A+e^{i z} \Pi^{\infty}$ in $\Pi^{\infty} / e^{i z} \Pi^{\infty}$ there is an operator $T\left(A+e^{i z} \Pi^{\infty}\right)$ defined on $\Pi^{2} / e^{i z} \Pi^{2}$ by

$$
T\left(A+e^{i z} \Pi^{\infty}\right): F+e^{i z} \Pi^{2} \rightarrow A F+e^{i z} \Pi^{2} .
$$

If $A \in H^{\infty}, T\left(A+e^{i z} \Pi^{\infty}\right)$ is unitarily equivalent to $T\left(A+e^{i z} H^{\infty}\right)$. Using this observation we note that the operator given by (1) is unitarily equivalent to the operator $T\left(K+e^{i z} \Pi^{\infty}\right)$, where

$$
K(z)=\int_{0}^{1} k(t) e^{i t z} d t .
$$

Hence the study of operators of the form (1) can be subsumed in the study of operators of the form (3). Any representative of the coset $K+e^{i z} \Pi^{\infty}$ will be called a symbol for $T$.

Operators of the form (3) are naturally intertwined by composition type operators. This idea was developed in some detail in [9]. Since it is used throughout the present paper, we sketch the main ideas and results.

Let $\Omega$ denote the class of functions $\theta(z)$ analytic in a half plane $y>\eta$, where $\eta$ is a real number depending on $\theta(z)$, and there satisfying the condition $\operatorname{Im} \theta(z)>y-\alpha$ for some real constant $\alpha$. We identify two elements of $\Omega$ if one is an analytic continuation of the other.

The following two results are proved in [9].

THEOREM 1. (i) For each $\theta \in \Omega$ there is a bounded operator $X_{\theta}$ on $\Pi^{2} / e^{i z} \Pi^{2}$ given by

$$
X_{\theta}: F+e^{i z} \Pi^{2} \rightarrow(\theta(z)+i) F(\theta(z)) /(z+i)+e^{i z} \Pi^{2} .
$$

(ii) If $\phi, \psi \in \Omega$, then $\phi \circ \psi \in \Omega$ and $X_{\phi \circ \psi}=X_{\psi} X_{\phi}$.

(iii) If $\phi, \psi \in \Omega$, then $X_{\phi}=X_{\psi}$ iff $1 / \phi-1 / \psi \in e^{i z} \Pi^{\infty}$.

THEOREM 2. Let $A, C \in \Pi^{\infty}$, and let $\theta \in \Omega$. Then $X_{\theta} T\left(C+e^{i z} \Pi^{\infty}\right)=$ $T\left(A+e^{i z} \Pi^{\infty}\right) X_{\theta}$ iff $C(\theta(z))-A(z) \in e^{i z} \Pi^{\infty}$.

The next result is a slight generalization of Theorem 6 of [9]. The proof is quite similar, and is omitted.

THEOREM 3. Let $\theta(z)=z+\chi(z)$, where $\chi(z)$ is analytic in some half plane $y>\eta$, and there satisfies the following conditions:

(i) $\operatorname{Im} \chi(z)$ is bounded.

(ii) $\chi(z)=o(|z|)$ as $z \rightarrow \infty$,

(iii) $\left|\chi^{\prime}(z)\right| \leqslant \rho$ for some $0 \leqslant \rho<1$.

Then $\theta(z)$ is univalent for $y>\eta, \theta, \theta^{-1} \in \Omega$, and $X_{\theta}$ is invertible with inverse $X_{\theta-1}$. 
Note that any function $\chi \in \Pi^{\infty}$ satisfies conditions (i)-(iii). (See [9].) An example of a function $\chi \notin \Pi^{\infty}$ which satisfies (i)-(iii) is $\chi(z)=\log z$. There are many others.

Combining Theorems 2 and 3, we obtain at once the following

Similarity Criterion. Let $A, C \in \Pi^{\infty}$. In order that $T\left(A+e^{i z} \Pi^{\infty}\right)$ and $T\left(C+e^{i z} \Pi^{\infty}\right)$ be similar, it suffices that there exist a function $\theta(z)$ satisfying the hypotheses of Theorem 3 such that $A(z)=C(\theta(z))$ in some half plane $y>\eta$.

2. Some classes of functions. By the similarity criterion we have just stated, we see that it is possible to establish similarity between two finite convolution operators by exhibiting a solution to a functional equation involving symbols for the operators. In the sequel, we shall derive conditions under which the desired solution will exist. Our first concern is with the following general question. Given an analytic function $f$ defined in a region $R$ and an analytic function $g$ defined in a simply connected region $R_{0}$, with $g\left(R_{0}\right) \subseteq f(R)$, what conditions are needed to insure the existence of an analytic function $h(z)$ such that $f(h(z))=$ $g(z)$ for all $z \in R_{0}$ ? In the language of algebraic topology, this question is known as the lifting problem (see Spanier [26, pp. 74-78]), and $h$ is called a lifting of $g$ (relative to $f$ ). Let us first note that if we make the assumption that $f$ is locally conformal (i.e., $f^{\prime}$ is nonvanishing), then a solution $h(z)$ will always exist locally in a neighborhood of every point of $R_{0}$. We are then reduced to finding additional conditions which will insure that the local solutions can be pieced together to form a global solution. We shall see that it suffices to assume that $f(z)$ is proper, i.e., $f^{-1}(K)$ is compact whenever $K$ is a compact subset of $f(R)$. Specifically, we have

LEMMA 3. Let $R$ be a region, and $f(z)$ a proper, locally conformal analytic function defined in $R$. Let $R_{0}$ be a simply connected region, and let $g(z)$ be an analytic function in $R_{0}$ with $g\left(R_{0}\right) \subseteq f(R)$. Let $z_{0} \in R_{0}$, and let $f_{0}^{-1}$ be any initial branch of $f^{-1}$ at $g\left(z_{0}\right)$. Then $f_{0}^{-1} \circ g$ continues to a single-valued analytic function in $R_{0}$.

Proof. Utilizing a theorem of Ahlfors and Sario [2, p. 29, Theorem 14F], one may easily show that $f_{0}^{-1}$ can be continued indefinitely along all arcs in $f(R)$, hence in particular along all arcs in $g\left(R_{0}\right)$. Thus $f_{0}^{-1} \circ g$ can be continued indefinitely along all arcs in $R_{0}$. Since $R_{0}$ is simply connected, the result now follows at once from the monodromy theorem.

Suppose now that we have a function $C(z)$ which is analytic and nonvanishing in a closed half plane $y \geqslant \eta$, and there satisfies $C(z) \rightarrow 0$ as $z \rightarrow \infty$. (As usual, a function is analytic in a closed set if it is analytic in a region containing the closed set.) It is easily shown that any such function is proper. Thus if $C(z)$ is in addition locally conformal, and if $A(z)$ is an analytic function mapping a half 
plane $y>\eta^{\prime}$ into the range of $C(z)$, it follows from Lemma 3 that there is an analytic function $\theta(z)$ defined for $y>\eta^{\prime}$ such that $C(\theta(z))=A(z)$ for $y>\eta^{\prime}$.

We shall henceforth assume that $C(z)$ satisfies the conditions laid down in the previous paragraph. Given another function $A \in \Pi^{\infty}$, to show that $T\left(A+e^{i z} \Pi^{\infty}\right)$ is similar to $T\left(C+e^{i z} \Pi^{\infty}\right)$, we first demonstrate that $A(z)$ maps some half plane $y>\eta^{\prime}$ into the range of $C(z)$. We will then be guaranteed the existence of a function $\theta(z)$ satisfying $C(\theta(z))=A(z)$ for $y>\eta^{\prime}$, and thus similarity will be established if it can be shown that $\theta(z)-z \in \Pi^{\infty}$. We shall see that this principle can be used to obtain simple sufficient conditions for similarity. However, it is first necessary to impose some additional structure on the functions $C(z)$ that we shall be considering. We begin with

Definition 1. A function $G(z)$ is said to belong to the class $G$ if it is analytic in a closed half plane $x \geqslant \eta$, for some $\eta>0$, and there satisfies the following conditions:

(1 $\left.{ }^{\circ}\right) G^{\prime}(z)$ is nonvanishing,

$\left(2^{\circ}\right) G^{\prime}(z) \rightarrow 0$ as $z \rightarrow \infty$,

(3) $G(x)>0$ for $x \geqslant \eta$,

$\left(4^{\circ}\right) \operatorname{Re} G(z) \rightarrow \infty$ as $z \rightarrow \infty$.

Two such functions are regarded as being identical if one is an analytic continuation of the other.

We remark that if $G \in G$, then $C(z)=\exp (-G(-i z))$ is nonvanishing and locally conformal in a closed half plane $y \geqslant \eta$, and there satisfies the condition $C(z) \rightarrow 0$ as $z \rightarrow \infty$. Condition $\left(2^{\circ}\right)$ provides control on the logarithmic derivative of $C$, and will be needed later. Condition $\left(3^{\circ}\right)$ insures that $C$ satisfies the reflection principle relative to the $y$-axis. While not totally indispensable, this will greatly simplify many of the arguments that follow.

For reference, we note two conspicuous properties of $G$. The proofs of these are elementary, and are left to the reader.

(i) If $\alpha>0$ and $G \in G$, then $\alpha G \in G$.

(ii) If $F, G \in G$ and $\left|F^{\prime}\right| G^{\prime} \mid \leqslant \rho$ for some $0<\rho<1$, then $F+G \in G$.

REMARK. In connection with (ii), we do not know if the sum of any two functions in $G$ lies in $G$. The trouble arises in trying to verify condition $\left(1^{\circ}\right)$.

The class $G$ will be the basic setting for all that follows. However, for technical reasons which will become apparent later on, we need an additional refinement. This leads us to

DEFINITION 2. Let $\Phi$ be a nonempty class of functions satisfying the following conditions:

(i) Each $\phi \in \Phi$ is defined in a closed half plane $x \geqslant \delta$, for some $\delta>0$.

(ii) Each $\phi \in \Phi$ satisfies $\phi(z) \rightarrow \infty$ as $z \rightarrow \infty$.

(iii) Each $\phi \in \Phi$ satisfies $\operatorname{Re} \phi(x) \rightarrow \infty$ as $x \rightarrow \infty$. 
A function $G(z)$ is said to belong to the class $G(\Phi)$ if $G \in G$ and satisfies the following additional condition:

$\left(5^{\circ}\right)$ For each $\phi \in \Phi$ there exists $\epsilon>0$ sufficiently small and a constant $M>0$ such that $\left|\operatorname{Re} G\left(z_{1}\right)-\operatorname{Re} G\left(z_{2}\right)\right| \leqslant \epsilon$ implies that $\left|G^{\prime}\left(\phi\left(z_{1}\right)\right) / G^{\prime}\left(z_{2}\right)\right| \leqslant M$ whenever $z_{1}$ and $z_{2}$ lie in some appropriately chosen half plane $x \geqslant \eta$.

Any class of functions $\Phi$ satisfying conditions (i)-(iii) will be called an asymptotic structure class, or simply an asymptotic structure.

REMARK. In connection with $\left(5^{\circ}\right)$, observe that condition (iii) insures that the composite $G^{\prime}(\phi(z))$ always makes sense in some half plane $x \geqslant \eta$.

The reader should note that the functions in an asymptotic structure class need not be analytic, or even continuous. As the name suggests, we are concerned not so much with the continuity properties of functions in such a class, but rather with their asymptotic behavior as $z \rightarrow \infty$.

At first glance the classes $G(\Phi)$ would seem to be rather exotic on account of the peculiar condition $\left(5^{\circ}\right)$. However, as the next result shows, these classes actually exhaust the class $G$. The importance of condition $\left(5^{\circ}\right)$ will become clear later on.

THEOREM 4. Let $G \in G$. Then there exists a function $\phi$ satisfying conditions (i)-(iii) of Definition 2, such that $G$ satisfies $\left(5^{\circ}\right)$ relative to $\phi$. Thus any function of class $G$ lies in $G(\Phi)$ for some asymptotic structure $\Phi$.

Proof. Fix some $\epsilon>0$, and choose $\eta>0$ so that $G$ satisfies $\left(1^{\circ}\right)-\left(4^{\circ}\right)$ for $x \geqslant \eta$. For each $z$ in this half plane, let $E(z)$ denote the set of all points $w$ in the half plane $x \geqslant \eta$ satisfying $|\operatorname{Re} G(z)-\operatorname{Re} G(w)| \leqslant \epsilon$. Observe that $E(z)$ is a compact set for each $z$ in the half plane $x \geqslant \eta$. For any such $z$, choose $z_{\text {min }} \in E(z)$ such that $\left|G^{\prime}\left(z_{\min }\right)\right|=\inf _{E(z)}\left|G^{\prime}(w)\right|$, and let $\phi(z)=z_{\text {min }}$. It is easily shown that the function $\phi$ so defined has the desired properties.

3. Asymptotic argument functions. Given $C(z)=\exp (-G(-i z))$ with $G \in$ G, and given $A \in \Pi^{\infty}$, to show that $T\left(A+e^{i z} \Pi^{\infty}\right)$ is similar to $T\left(C+e^{i z} \Pi^{\infty}\right)$ by the method we have outlined, it is first necessary to prove that $A(z)$ maps some half plane $y>\eta^{\prime}$ into the range of $C(z)$. While there are many ways of accomplishing this, perhaps the simplest is to show that the range of $C$ contains some particularly simple region, and that $A$ maps a half plane $y>\eta^{\prime}$ into this region. This is the course that we shall follow. Specifically, we shall determine conditions under which the range of $C$ contains a punctured disk centered at 0 . The function $A(z)$ will generally have the form $A(z)=C(z)[1+o(1)]$ as $z \rightarrow \infty$. Note that a function of this form can always be made to map some half plane $y>\eta^{\prime}$ into any preassigned punctured disk centered at 0 . Thus if the range of $C$ contains a punctured disk centered at $0, A$ will map some half plane $y>\eta^{\prime}$ into the range of $C$. 
Given a function $C(z)=\exp (-G(-i z)), G \in G$, we shall see that whether or not the range of $C$ contains a punctured disk centered at 0 is essentially controlled by the asymptotic behavior of $\arg C(z)$ as $z \rightarrow \infty$. It is therefore advantageous to develop some terminology for dealing with this phenomenon. It is agreed that we shall always choose the determination of arg $C(z)$ which vanishes on the imaginary axis; i.e., we choose $\arg C(z)=-\operatorname{Im} G(-i z)$. We begin by obtaining two simple lemmas.

LEMma 4. If $G \in G$ then $\operatorname{Im} G(z) \rightarrow 0$ uniformly as $z \rightarrow \infty$ in any strip $-a \leqslant y \leqslant a, a>0$.

Proof. Since $G^{\prime}(z) \rightarrow 0$ as $z \rightarrow \infty$ in some closed half plane $x \geqslant \eta$, in particular $G^{\prime}(z) \rightarrow 0$ as $x \rightarrow \infty$. Thus for any $\epsilon>0$ we may choose $M>0$ such that $x>M$ implies $\left|G^{\prime}(z)\right|<\epsilon / a$. Thus if $x>M$ and $-a \leqslant y \leqslant a$, we have

$$
\begin{aligned}
\operatorname{Im} G(z) \mid & =\left|\operatorname{Im} \int_{\eta}^{z} G^{\prime}(w) d w\right|=\left|\operatorname{Re} \int_{0}^{y} G^{\prime}(x+i t) d t\right| \\
& \leqslant \int_{0}^{|y|}\left|G^{\prime}(x+i t)\right| d t<\epsilon .
\end{aligned}
$$

The lemma follows.

LEMMA 5. Let $G \in G$. Then for any $0 \leqslant \theta \leqslant \pi / 2$, and for any $\eta$ and $\eta^{\prime}$ sufficiently large, $\operatorname{Im}\left[G\left(\eta+r e^{i \theta}\right)-G\left(\eta^{\prime}+r e^{i \theta}\right)\right] \rightarrow 0$ as $r \rightarrow \infty$.

The proof of this is similar to the proof of the previous lemma, and is left to the reader.

Now if $G \in G$ and $C(z)=\exp (-G(-i z))$, then by the reflection principle we have

$$
\arg C(-x+i y)=-\operatorname{Im} G(y+i x)=\operatorname{Im} G(y-i x)=-\arg C(x+i y) .
$$

Thus we see that all the information that can be obtained about arg $C(z)$ is contained in its behavior in the second quadrant, or what is the same thing, the behavior of $\operatorname{Im} G(z)$ in the first quadrant.

We shall be concerned with the asymptotic behavior of $\operatorname{Im} G(z)$ on a ray $z=\eta+r e^{i \theta}, r>0$, as $r \rightarrow \infty$. In view of the foregoing remarks, it will suffice to restrict $\theta$ to the range $0 \leqslant \theta \leqslant \pi / 2$. Motivated by this, we make the following

Definition 3. Let $G \in G$, and let $C(z)=\exp (-G(-i z))$. Let $\eta>0$ be chosen so that $G$ satisfies $\left(1^{\circ}\right)-\left(4^{\circ}\right)$ for $x \geqslant \eta$. We define the upper and lower asymptotic argument functions of $C(z)$ by

$$
\begin{aligned}
& h^{+}(\theta ; C)=\limsup _{r \rightarrow \infty}\left|\operatorname{Im} G\left(\eta+r e^{i \theta}\right)\right|, \\
& h^{-}(\theta ; C)=\liminf _{r \rightarrow \infty}\left|\operatorname{Im} G\left(\eta+r e^{i \theta}\right)\right|,
\end{aligned}
$$


$0 \leqslant \theta \leqslant \pi / 2$. In cases when $h^{-}(\theta ; C) \equiv h^{+}(\theta ; C)$, we denote the common function by $h(\theta ; C)$, which will simply be called the asymptotic argument function of $C(z)$.

REMARK. Observe that on account of Lemma 5 , the functions $h^{+}(\theta ; C)$ and $h^{-}(\theta ; C)$ are independent of $\eta$, in the sense that equation (6) remains unchanged if $\eta$ is replaced by any $\eta^{\prime} \geqslant \eta$.

We wish to stress the fact that $h^{+}(\theta ; C)$ and $h^{-}(\theta ; C)$ are extended real valued functions. Indeed, as we shall see, in many interesting applications $h^{-}(\theta ; C)=h^{+}(\theta ; C)=+\infty$ for $0<\theta \leqslant \pi / 2$.

The next result summarizes the most useful properties of the functions $h^{ \pm}(\theta ; C)$.

Theorem 5. Let $G \in G$ and let $C(z)=\exp (-G(-i z))$. Then

(i) $h^{-}(\theta ; C) \leqslant h^{+}(\theta ; C)$ for all $\theta$,

(ii) $h^{+}(\theta ; C)$ is a nondecreasing function of $\theta$,

(iii) $h^{ \pm}\left(\theta ; C^{\alpha}\right)=\alpha h^{ \pm}(\theta ; C)$ for any $\alpha>0$.

Before proving this result, we shall need some preliminary notions. First let us observe that the function $C(z)$ maps some half plane $y \geqslant \eta$ into a punctured disk centered at 0 , which we may assume without loss of generality to have unit radius. We denote this punctured disk by $D_{0}$. Let $\hat{D}_{0}$ denote the universal covering space of $D_{0}$, and pr the canonical projection of $\hat{D}_{0}$ onto $D_{0}$. We may think of $\hat{D}_{0}$ as the strip $\{(r, \theta) \mid 0<r<1,-\infty<\theta<\infty\}$. Then $\operatorname{pr}(r, \theta)=r e^{i \theta}$. As usual, $\hat{D}_{0}$ is given the conformal structure induced by the local action of pr. Now if $f(z)$ is any analytic function mapping a simply connected region $R$ into $D_{0}$, it is well known that $f(z)$ can be lifted to an analytic map $\hat{f}(z)$ from $R$ to $\hat{D}_{0}$; i.e., there is an analytic map $\hat{f}$ from $R$ to $\hat{D}_{0}$ such that $\mathrm{pr} \circ \hat{f}=f$. Specifically, we may choose $\hat{f}(z)=(|f(z)|$, arg $f(z))$, for any initial determination of arg $f(z)$. In particular, $C(z)$ has such a lifting $\hat{C}(z)$, where, in this case, the determination of $\arg C(z)$ is as stipulated above.

In the sequel, we shall make use of the following notation. For any $\eta>0$ and $0<\beta<1$, we denote by $R(\eta, \beta)$ the closed sector $\{z \mid z=\eta$ or $|\arg (-i z-\eta)|$ $\leqslant \beta \pi\}$. We are now ready for the

Proof of Theorem 5. The only nontrivial part is (ii). The rest is left to the reader. To prove (ii), suppose to the contrary that $\theta_{1}\left\langle\theta_{2}\right.$ and $\left.h^{+}\left(\theta_{1} ; C\right)\right\rangle$ $h^{+}\left(\theta_{2} ; C\right)$. Choose $\epsilon>0$ such that $h^{+}\left(\theta_{1} ; C\right)-h^{+}\left(\theta_{2} ; C\right)>\epsilon$, and let $N>0$ be chosen sufficiently large so that $|\arg (-i z-\eta)|=\theta_{2}$ and $|z|>N$ imply that $|\arg C(z)|<h^{+}\left(\theta_{2} ; C\right)+\epsilon$. Since $C(z)$ is nonvanishing, we may choose $\rho>0$ sufficiently small so that $|C(z)| \geqslant \rho$ for $|z| \leqslant N$. Now choose $N^{\prime}>0$ sufficiently large so that $|z|>N^{\prime}$ implies $|C(z)|<\rho$. Choose some $z_{0}$ with $\left|\arg \left(-i z_{0}-\eta\right)\right|$ $=\theta_{1}$ and $\left|z_{0}\right|>N^{\prime}$ such that $\operatorname{larg} C\left(z_{0}\right) \mid>h^{+}\left(\theta_{2} ; C\right)+\epsilon$. On account of (5) 
there is no generality lost in assuming that $\arg C\left(z_{0}\right)>0$. Let $L=\{(r, \theta) \mid r=$ $\left|C\left(z_{0}\right)\right|$ and $\left.0 \leqslant \theta<\infty\right\}$. Now if $|\arg (-i z-\eta)|=\theta_{2}$ and $\hat{C}(z) \in L$, then necessarily $|z|>N$, so that $\arg C(z)<\arg C\left(z_{0}\right)$. But now choose some $z^{\prime} \in$ $R\left(\eta, \theta_{2} / \pi\right)$ such that $\hat{C}\left(z^{\prime}\right) \in L$ and $\arg C\left(z^{\prime}\right)$ is maximum among all such points. By the open mapping principle (applied to $\hat{C}$ ), necessarily $z^{\prime} \in \partial R\left(\eta, \theta_{2} / \pi\right)$.

Hence by what was just proved, $\arg C\left(z^{\prime}\right)<\arg C\left(z_{0}\right)$, which plainly contradicts the maximality of $\arg C\left(z^{\prime}\right)$. This contradiction establishes the result.

4. A similarity theorem. In this section, we shall prove the following fundamental result.

THEOREM 6. Let $G \in G(\Phi)$ for some asymptotic structure $\Phi$, and let $C(z)$ $=\exp (-G(-i z))$. Assume that $h^{-}(\pi / 2 ; C)>0$. Let $A \in \Pi^{\infty}$ be of the form

$$
A(z)=C(z)[1+P(z)],
$$

where $P(z)=O\left(\left|G^{\prime}(\phi(-i z))\right|\right)$ as $z \rightarrow \infty$ in some closed half plane $y \geqslant \eta$, and $\phi \in \Phi$. Then $T\left(A+e^{i z} \Pi^{\infty}\right)$ is similar to $T\left(C+e^{i z} \Pi^{\infty}\right)$.

Note that since $G^{\prime}(\phi(z)) \rightarrow 0$ as $z \rightarrow \infty, P(z) \rightarrow 0$ as $z \rightarrow \infty$. Thus in particular (7) asserts that $A(z)=C(z)[1+o(1)]$ as $z \rightarrow \infty$.

Intuitively speaking, this result offers support to the feeling that operators of the form (1) will divide themselves into similarity classes according to the asymptotic behavior of their symbols as $z \rightarrow \infty$. In the face of Tauberian intuition, this in turn provides evidence in favor of the idea that operators of the form (1) should somehow be partitioned into similarity classes according to the behavior of their kernels in a neighborhood of 0 .

Before proceeding with the proof of Theorem 6, we introduce some additional notation. Let $D_{0}$ be the punctured unit disk centered at 0 . If $z_{1}=r_{1} e^{i \theta_{1}}$ and $z_{2}=r_{2} e^{i \theta_{2}}$ are in $D_{0}$, we denote by $\Gamma\left(z_{1}, z_{2}\right)$ the curve consisting of the shorter of the two arcs of the circle $|w|=r_{1}$ joining the points $z_{1}$ and $r_{1} e^{i \theta_{2}}$ followed by the segment of the ray arg $w=\theta_{2}$ joining $r_{1} e^{i \theta_{2}}$ and $z_{2}$. Since we shall only be concerned with the case $\left|\theta_{1}-\theta_{2}\right|<\pi$, the curve $\Gamma\left(z_{1}, z_{2}\right)$ is unambiguously defined. Elementary estimates show that there is a constant $\lambda>0$ such that $\left|\Gamma\left(z_{1}, z_{2}\right)\right| \leqslant \lambda\left|z_{1}-z_{2}\right|$ for any $z_{1}, z_{2} \in D_{0}$. (Here $\left|\Gamma\left(z_{1}, z_{2}\right)\right|=$ length of $\Gamma\left(z_{1}, z_{2}\right)$.) We are now ready for the

Proof of Theorem 6. We first consider the case $h^{-}(\pi / 2 ; C)>\pi$. By hypothesis, the range of $C(z)$ is contained in a punctured disk centered at 0 , which we may with no loss of generality choose to be $D_{0}$. As above, $\hat{D}_{0}$ will denote the universal covering space of $D_{0}$, and $\hat{C}(z)$ the lifting of $C(z)$ to $\hat{D}_{0}$. Since $h^{-}(\pi / 2 ; C)>\pi$, we may choose $N>0$ such that $|\arg (-i z-\eta)|=\pi / 2$ and $|z|>N$ imply that $|\arg C(z)|>\pi$. Also there is a $\rho>0$ sufficiently small so 
that $|C(z)| \geqslant \rho$ for $|z| \leqslant N$. It then follows from the open mapping principle that the range of $\hat{C}(\mathrm{z})$ contains the rectangle $\{(r, \theta) \mid 0<r<\rho,-\pi \leqslant \theta \leqslant \pi\}$. Thus the range of $C(z)$ contains the punctured disk $D_{\rho}=\{w|0<| w \mid<\rho\}$.

By using (7) we may choose $\eta^{\prime}>0$ sufficiently large so that the following conditions are satisfied:

(i) $A(z)$ is nonvanishing for $y>\eta^{\prime}$,

(ii) $A(z)$ and $C(z)$ both map the half plane $y>\eta^{\prime}$ into $D_{\rho}$,

(iii) $|\arg C(z)-\arg A(z)|<\pi$ for $y>\eta^{\prime}$,

(iv) $|P(z)| \leqslant \delta=\min \left\{e^{\epsilon}-1,1-e^{-\epsilon}\right\}$, where $\epsilon$ is as in $\left(5^{\circ}\right)$.

Now by hypothesis $C(z)$ is locally conformal and, as was previously observed, proper. Hence by the proof of Lemma 3, any initial branch of $C^{-1}$ can be continued indefinitely along all arcs in the range of $C(z)$. Let $z_{0}$ be a point in the half plane $y>\eta^{\prime}$, and let $C_{0}^{-1}$ be the initial branch of $C^{-1}$ at $C\left(z_{0}\right)$ such that $C_{0}^{-1}(C(z))=z$ in a neighborhood of $z_{0}$. Continue $C_{0}^{-1}$ analytically along $\Gamma\left(C\left(z_{0}\right), A\left(z_{0}\right)\right)$ to $A\left(z_{0}\right)$, and denote the terminal branch so obtained by $C_{1}^{-1}$. Let $\theta(z)=C_{1}^{-1}(A(z))$ in some neighborhood of $z_{0}$. By Lemma 3, $\theta(z)$ continues analytically throughout the half plane $y>\eta^{\prime}$ to a solution of the equation $C(\theta(z))=A(z)$. It remains to be shown that $\theta(z)-z \in \Pi^{\infty}$.

Fix $z$ in the half plane $y>\eta^{\prime}$. By (iii) above, $C(z)$ and $A(z)$ lie in a sector $\Sigma$ of angle $<\pi$. Choose the branch $C_{z}^{-1}$ of $C^{-1}$ at $A(z)$ such that $C_{z}^{-1}(A(z))=$ $\theta(z)$. By the monodromy theorem, $C_{z}^{-1}$ continues analytically to a single-valued function in $\Sigma$. Moreover, the monodromy theorem also implies that $C_{z}^{-1}(C(z))$ $=z$.

Now from (7) we have

Hence the curve $\gamma=\Gamma(C(z), A(z))$ lies entirely within the annulus $\{w|(1-\delta)| C(z) \mid$ $\leqslant|w| \leqslant(1+\delta)|C(z)|\}$, Now we have

$$
|\theta(z)-z|=\left|C_{z}^{-1}(A(z))-C_{z}^{-1}(C(z))\right|=\left|\int_{\gamma}\left(C_{z}^{-1}\right)^{\prime}(w) d w\right|
$$

$$
\leqslant\left|\left(C_{z}^{-1}\right)^{\prime}\left(w_{1}\right) \| \gamma\right|
$$

for some $w_{1} \in \gamma$. Let $z_{1}=C_{z}^{-1}\left(w_{1}\right)$. Then from (9) we have

$$
\begin{aligned}
|\theta(z)-z| & \leqslant \lambda|A(z)-C(z)| /\left|C^{\prime}\left(z_{1}\right)\right| \\
& =\lambda|C(z) P(z)| /\left|C^{\prime}\left(z_{1}\right)\right| \\
& \leqslant \lambda K\left|C(z)\left\|G^{\prime}(\phi(-i z))|/| C\left(z_{1}\right)\right\| G^{\prime}\left(-i z_{1}\right)\right|,
\end{aligned}
$$

where $K>0$ is a constant independent of $z$. But since

$$
(1-\delta)|C(z)| \leqslant\left|C\left(z_{1}\right)\right| \leqslant(1+\delta)|C(z)|
$$


we have from (10) that

$$
|\theta(z)-z| \leqslant \lambda K(1-\delta)^{-1}\left|G^{\prime}(\phi(-i z))\right| /\left|G^{\prime}\left(-i z_{1}\right)\right| .
$$

But then on account of $\left(5^{\circ}\right)$ we obtain

$$
|\theta(z)-z| \leqslant \lambda K M(1-\delta)^{-1},
$$

where $M$ is as in $\left(5^{\circ}\right)$. Since $z$ was an arbitrary point in the half plane $y>\eta^{\prime}$, it follows that $\theta(z)-z \in \Pi^{\infty}$. The result is therefore established in the case $h^{-}(\pi / 2 ; C)>\pi$.

Now suppose that $0<h^{-}(\pi / 2 ; C) \leqslant \pi$. By Theorem 5 (iii) we may choose an integer $n$ sufficiently large so that $h^{-}\left(\pi / 2 ; C^{n}\right)>\pi$. Note that

$$
A(z)^{n}=C(z)^{n}[1+P(z)]^{n}=C(z)^{n}\left[1+O\left(\left|G^{\prime}(\phi(-i z))\right|\right)\right] .
$$

Thus by the first part of the proof we can find $\theta(z)$ with $\theta(z)-z \in \Pi^{\infty}$ such that $C(\theta(z))^{n}=A(z)^{n}$ in some half plane $y>\eta^{\prime}$. Thus for $y>\eta^{\prime}$ we have $C(\theta(z))=$ $\xi A(z)$, where $\xi$ is some $n$th root of unity. We will be finished if we can show that $\xi=1$. But in particular

$$
\arg \xi=\arg C(\theta(i y))-\arg A(i y)
$$

for $y>\eta^{\prime}$. As $y \rightarrow \infty$, $\arg A(i y) \rightarrow 0$ by (7) and $\arg C(\theta(i y)) \rightarrow 0$ by Lemma 4. The result follows.

It is clear from the proof of Theorem 6 that if $C(z)=\exp (-G(-i z)), G \in$ $G$, and $h^{-}(\pi / 2 ; C)>0$, then in order to obtain a function $\theta(z)$ such that $C(\theta(z))$ $=A(z)$ holds in some half plane $y>\eta^{\prime}$, it suffices that $A(z)=C(z)[1+o(1)]$ as $z \rightarrow \infty$. However, to say anything more about $\theta(z)$, one must put some limitation on the $o(1)$-term. (This is aptly demonstrated by the example of Kalisch [17].) As the proof of the theorem indicates, the classes $G(\Phi)$ were introduced to expedite this purpose. In a practical application, the main difficulty lies in showing that the class $\Phi$ can be chosen to contain some particularly tractable function or family of functions. The remainder of this paper is dedicated to examining some special cases in which this difficulty can be surmounted.

5. Functions of regular type. Let $G(z)$ be analytic in a half plane $x \geqslant \eta$, for some $\eta>0$. As before, we set $C(z)=\exp (-G(-i z))$. If $0 \leqslant \alpha<1$, we say that $G(z)$ is of regular type $\alpha$ if $G^{\prime}(z)=z^{\alpha-1}[\beta+\epsilon(z)]$, where $\beta>0, \epsilon(z) \rightarrow 0$ as $z \rightarrow \infty$ in the half plane $x \geqslant \eta$, and $\epsilon(x)$ is real for $x \geqslant \eta$. We begin with the following basic result.

THEOREM 7. Let $G(z)$ be of regular type $\alpha$ for some $0 \leqslant \alpha<1$. Then $G \in G$. 
Proof. It is easy to check that $G$ satisfies $\left(1^{\circ}\right)-\left(3^{\circ}\right)$ in some half plane $x \geqslant \eta$. To show that $\left(4^{\circ}\right)$ is satisfied, the cases $\alpha=0$ and $\alpha>0$ must be distinguished.

Suppose $\alpha=0$. Choose $\eta^{\prime}>0$ sufficient'y large so that $|\epsilon(z)| \leqslant \beta_{0}<\beta$ for $x \geqslant \eta^{\prime}$. Then for $x \geqslant \eta^{\prime}$ we have

$$
\begin{aligned}
\operatorname{Re} G(z) & =\beta \operatorname{Re} \int_{\eta^{\prime}}^{z} d w / w+\operatorname{Re} \int_{\eta^{\prime}}^{z} \epsilon(w) d w / w+G\left(\eta^{\prime}\right) \\
& \geqslant \beta \log |z|-\beta \log \eta^{\prime}+G\left(\eta^{\prime}\right)-\left|\int_{\eta^{\prime}}^{z} \epsilon(w) d w / w\right| \\
& \geqslant \beta \log |z|-\beta \log \eta^{\prime}+G\left(\eta^{\prime}\right)-\int_{\eta^{\prime}}^{|z|}|\epsilon(t)| d t / t-\int_{0}^{\arg z \mid}\left|\epsilon\left(|z| e^{i \theta}\right)\right| d \theta \\
& \geqslant\left(\beta-\beta_{0}\right) \log |z|-\left(\beta-f_{0}\right) \log \eta^{\prime}+G\left(\eta^{\prime}\right)-\beta_{0} \operatorname{larg} z \mid .
\end{aligned}
$$

Thus we see that $\operatorname{Re} G(z) \rightarrow \infty$ as $z \rightarrow \infty$ in the half plane $x \geqslant \eta^{\prime}$, as desired.

The case $\alpha>0$ is handled similarly.

In view of this result, we are in a position to apply Theorem 6 to the function $C(z)=\exp (-G(-i z))$ whenever $G(z)$ is of regular type $\alpha$ for some $0 \leqslant \alpha<1$. To accomplish this, we must establish that $C(z)$ satisfies the condition $h^{-}(\pi / 2 ; C)$ $>0$, and determine an appropriate asymptotic structure class $\Phi$ such that $G \in$ $G(\Phi)$. As we shall see, in this case $\Phi$ can always be taken to consist of particularly tractable functions. The importance of functions of regular type is aptly demonstrated by the frequency with which they arise in connection with concrete examples of operators of the form (1). (See below.)

As can be seen from the proof of Theorem 7, the cases $\alpha=0$ and $\alpha>0$ tend to require separate treatment. We shall see the gap between these two cases widen much farther in what follows. Consequently, we devote a separate section to each of them

6. Slowly varying functions. In this section we treat the case $\alpha=0$. Essentially, this case has previously been considered in [9]. Here we are primarily concerned with rephrasing the results of [9] in terms of Theorem 6. Let us first recall from [9] that a function $L(z)$ analytic and nonvanishing for $x \geqslant \eta$, with $L(x)$ $>0$ for $x \geqslant \eta$ and $z L^{\prime}(z) / L(z) \rightarrow 0$ as $z \rightarrow \infty$ in the half plane $x \geqslant \eta$ is said to be slowly varying at infinity in the half plane $x \geqslant \eta$. See also Feller [8, pp. 268276], Gohberg and Krein [12, pp. 144-145], and Levin [19, pp. 31-33].

The following lemma was proved in [9].

LEMMA 6. Let $L(z)$ be slowly varying at infinity in the half plane $x \geqslant \eta$. Then

(i) $|L(z)| / L(|z|) \rightarrow 1$ as $z \rightarrow \infty$ in the half plane $x \geqslant \eta$,

(ii) $\arg L(z) \rightarrow 0$ as $z \rightarrow \infty$ in the half plane $x \geqslant \eta$,

(iii) $L(t x) / L(x) \rightarrow 1$ as $x \rightarrow \infty$ for every $t>0$, 
(iv) for every $\epsilon>0$ there exists a constant $C(\epsilon)>0$ such that $C(\epsilon)^{-1}|z|^{-\epsilon}$ $\leqslant|L(z)| \leqslant C(\epsilon)|z|^{\epsilon}$ for all $z$ in the half plane $x \geqslant \eta$.

The next lemma provides the connection between slowly varying functions and functions of regular type 0 .

LEMMA 7. If $C(z)=\exp (-G(-i z))$, then $G(z)$ is of regular type 0 iff $C(z)$ $=L(-i z) /(-i z)^{\beta}$, where $\beta>0$ and $L(z)$ is slowly varying at infinity in some half plane $x \geqslant \eta$.

The proof of this is routine, and is left to the reader.

REMARK. It follows from this result that if $G(z)=\log (z L(z))$, where $L(z)$ is slowly varying at infinity in some half plane $x \geqslant \eta$, then $G \in G$.

Now if $C(z)=L(-i z) /(-i z)^{\beta}$, where $\beta>0$ and $L(z)$ is slowly varying at infinity in some half plane $x \geqslant \eta$, then

$$
\arg C(z)=\arg L(-i z)-\beta \arg (-i z) .
$$

We therefore have $h^{-}(\theta ; C)=h^{+}(\theta ; C)=\beta \theta$. Thus in particular the condition $h^{-}(\pi / 2 ; C)>0$ is satisfied for any such function $C(z)$.

Many of the slowly varying functions which arise in examples satisfy additional useful conditions. Specifically, if $L(z)$ is slowly varying at infinity in the half plane $x \geqslant \eta$, it often happens that $L(z)$ also satisfies

(i) $L(x)$ is ultimately monotone as $x \rightarrow \infty$,

(ii) $L\left(x^{p}\right) / L(x)$ remains bounded as $x \rightarrow \infty$, for any $p>0$.

Such a function will be called slowly varying at infinity in the strong sense.

Functions of this type were studied in [9], and in fact all the specific examples presented there were slowly varying at infinity in the strong sense. Perhaps the best explanation for this phenomenon is that all the specific examples listed in [9], and indeed most of the interesting classical examples, involve logarithms. We assert that in fact any such example is slowly varying at infinity in the strong sense. Specifically, define $\log ^{m} z$ for $m$ a positive integer by $\log ^{1} z=$ $\log z$ and $\log ^{m} z=\log \left(\log ^{m-1} z\right)$ for $m \geqslant 2$, and let

$$
L(z)=\sum_{k=1}^{n} c_{k}\left(\log ^{m} k 1 z\right)^{\rho} \rho_{1} \cdots\left(\log ^{m} k j z\right)^{\rho_{k j}},
$$

where the $c_{k}$ are positive constants, the $m_{k i}$ are positive integers, the $\rho_{k i}$ are real numbers, and $j$ is a positive integer. Such a function will simply be referred to as a logarithmic function. It is not difficult to check that any such function is slowly varying at infinity in some half plane $x \geqslant \eta$, and satisfies condition (ii) in the above definition. The fact that any such function satisfies the ultimate monotonicity requirement results from a theorem of Hardy [14, pp. 17-20]. Thus any logarithmic function is slowly varying at infinity in the strong sense. 
As we observed above, if $G(z)=\log (z L(z))$, where $L(z)$ is slowly varying at infinity in some half plane $x \geqslant \eta$, then $G \in G$. The next result provides some simple asymptotic structure classes $\Phi$ such that $G \in G(\Phi)$.

THEOREM 8. Let $L(z)$ be slowly varying at infinity in a half plane $x \geqslant \eta$, and let $G(z)=\log (z L(z))$. Then $G \in G(\Phi)$, where $\Phi=\left\{\phi(z)=|z|^{p}, p>1\right\}$. If in addition $L(z)$ is slowly varying at infinity in the strong sense, $G \in G\left(\Phi^{\prime}\right)$, where $\Phi^{\prime}=\{\phi(z)=z\}$.

Proof. It suffices to show that condition $\left(5^{\circ}\right)$ is verified for the given asymptotic structure classes.

We first assume only that $L(z)$ is slowly varying at infinity. Let $\epsilon>0$ be fixed, and let $z_{1}$ and $z_{2}$ be points of the half plane $x \geqslant \eta$ for which $\mid \operatorname{Re} G\left(z_{1}\right)-$ $\operatorname{Re} G\left(z_{2}\right) \mid \leqslant \epsilon$ holds. Then

$$
e^{-\epsilon} \leqslant\left|z_{2} L\left(z_{2}\right) / z_{1} L\left(z_{1}\right)\right| \leqslant e^{\epsilon} .
$$

For any $p>1$, choose $\delta>0$ such that $(1+\delta) /(1-\delta) \leqslant p$. By Lemma 6 (iv), we have

$$
\left|z_{2}\right|^{-\delta} \leqslant C(\delta)\left|L\left(z_{2}\right)\right|
$$

Thus by (15) we have

$$
\begin{aligned}
\left|z_{2}\right| & \leqslant C(\delta)^{1 /(1-\delta)}\left|z_{2} L\left(z_{2}\right)\right|^{1 /(1-\delta)} \\
& \leqslant e^{\epsilon /(1-\delta)} C(\delta)^{1 /(1-\delta)}\left|z_{1} L\left(z_{1}\right)\right|^{1 /(1-\delta)}
\end{aligned}
$$

Again using Lemma 6(iv), we have

$$
\left|L\left(z_{1}\right)\right| \leqslant C(\delta)\left|z_{1}\right|^{\delta}
$$

Therefore

$$
\begin{aligned}
\left|z_{1} L\left(z_{1}\right)\right|^{1 /(1-\delta)} & \leqslant C(\delta)^{1 /(1-\delta)}\left|z_{1}\right|^{(1+\delta) /(1-\delta)} \\
& \leqslant C(\delta)^{1 /(1-\delta)}\left|z_{1}\right|^{p}
\end{aligned}
$$

Using (17), we then have

$$
\left|z_{2}\right| \leqslant\left[e^{\epsilon} C(\delta)^{2}\right]^{1 /(1-\delta)}\left|z_{1}\right|^{p}
$$

From this it follows easily that $G \in G(\Phi)$.

Now assume $L(z)$ to be slowly varying at infinity in the strong sense. For definiteness, we suppose that $L(x)$ is ultimately nonincreasing. (The other case is treated similarly.) We have only to show that $G(z)$ satisfies $\left(5^{\circ}\right)$ relative to 
$\phi(z)=z$. If $\epsilon>0$ is fixed and $z_{1}$ and $z_{2}$ are points in the half plane $x$ for which $\left|\operatorname{Re} G\left(z_{1}\right)-\operatorname{Re} G\left(z_{2}\right)\right| \leqslant \epsilon$, we repeat the previous argument as far as (20), with some $p>1$ fixed. Now by Lemma 6(i), we have

$$
\left|L\left(z_{1}\right)\right| \leqslant K_{0} L\left(\left|z_{1}\right|\right),
$$

where $K_{0}>0$ is a constant. But since $L(z)$ is slowly varying at infinity in the strong sense, there is a constant $K_{1}>0$ such that

$$
K_{0} L\left(\left|z_{1}\right|\right) \leqslant K_{1} L\left(\left|z_{1}\right|^{p}\right) \text {. }
$$

Then by Lemma 6(iii), there is a constant $K_{2}>0$ such that

$$
K_{1} L\left(\left|z_{1}\right|^{p}\right) \leqslant K_{2} L\left(\left[e^{\epsilon} C(\delta)^{2}\right]^{1 /(1-\delta)}\left|z_{1}\right|^{p}\right) .
$$

Then since $L(x)$ is ultimately nonincreasing, we have from (20) that

$$
K_{2} L\left(\left[e^{\epsilon} C(\delta)^{2}\right]^{1 /(1-\delta)}\left|z_{1}\right|^{p}\right) \leqslant K_{3} L\left(\left|z_{2}\right|\right),
$$

where $K_{3}>0$ is a constant. But then using Lemma 6(i) again, we have

$$
K_{3} L\left(\left|z_{2}\right|\right) \leqslant K_{4}\left|L\left(z_{2}\right)\right|
$$

for some constant $K_{4}>0$. Combining (21)-(25), we obtain

$$
\left|L\left(z_{1}\right)\right| \leqslant K_{4}\left|L\left(z_{2}\right)\right|
$$

By (15) we then have

$$
\left|z_{2} / z_{1}\right| \leqslant K_{4} e^{\epsilon}
$$

From this the desired result follows easily.

Combining Theorem 8 and the remark following Lemma 7 with Theorem 6, we obtain at once the following result.

THEOREM 9. Let $L(z)$ be slowly varying at infinity in some half plane $x \geqslant$ $\eta$, and let $C(z)=L(-i z) /(-i z)^{\beta}$ for some $\beta>0$. Suppose that $A \in \Pi^{\infty}$, and that $A$ can be expressed in the form

$$
A(z)=C(Z)[1+P(z)],
$$

where $P(z)=O\left(1 /|z|^{p}\right)$ as $z \rightarrow \infty$ in some closed half plane $y \geqslant \eta^{\prime}$, and $p>1$. Then $T\left(A+e^{i z} \Pi^{\infty}\right)$ and $T\left(C+e^{i z} \Pi^{\infty}\right)$ are similar. If in addition $L(z)$ is slowly varying at infinity in the strong sense, it suffices that $P(z)=O(1 /|z|)$ as $z \rightarrow \infty$.

We remark that Theorem 9 is slightly stronger than Theorem 8 of [9], since it was there assumed that $L(z)$ was slowly varying at infinity in a sector properly containing a half plane. 
7. Regularly varying functions. In this section we treat the case $\alpha>0$. We are assuming that $G(z)$ is analytic in some half plane $x \geqslant \eta$, and that $G^{\prime}(z)=$ $z^{\alpha-1}[\alpha \beta+\epsilon(z)]$, where $0<\alpha<1, \beta>0$, and $\epsilon(z) \rightarrow 0$ as $z \rightarrow \infty$ in the half plane $x \geqslant \eta$, with $\epsilon(x)$ real for $x \geqslant \eta$. We begin with

Definition 4. Let $L(z)$ be analytic and nonvanishing in a half plane $x \geqslant$ $\eta, L(x)>0$ for $x \geqslant \eta$, and $z^{1-\alpha} L^{\prime}(z) / L(z) \rightarrow 0$ as $z \rightarrow \infty$ in the half plane $x \geqslant$ $\eta$, for some $0<\alpha<1$. Then $L(z)$ is said to be regularly varying at infinity of order $\alpha$ in the half plane $x \geqslant \eta$.

LEMMA 8. Let $0<\alpha<1$, and let $L(z)$ be a function regularly varying at infinity of order $\alpha$ in some half plane $x \geqslant \eta$. Then for any $\epsilon>0$ there exists $a$ constant $C(\epsilon)>0$ such that

$$
C(\epsilon)^{-1} e^{-\epsilon|z|^{\alpha}} \leqslant|L(z)| \leqslant C(\epsilon) e^{\epsilon|z|^{\alpha}}
$$

holds for all $z$ in the half plane $x \geqslant \eta$.

Proof. In view of the fact that the reciprocal of a function regularly varying at infinity of order $\alpha$ is also regularly varying at infinity of order $\alpha$, it suffices to prove the right-hand inequality. For any $\epsilon>0$ choose $N>0$ such that $|z|>$ $N$ implies that $\left|z^{1-\alpha} L^{\prime}(z) / L(z)\right|<2 \alpha \epsilon /(2+\alpha \pi)$. Then for $|z|>N$ we have

$$
\begin{aligned}
|L(z)| & \leqslant\left|\exp \left[\log L(N)+\int_{N}^{z} L^{\prime}(w) / L(w) d w\right]\right| \\
& \leqslant L(N) \exp \int_{N}^{|z|} \frac{L^{\prime}(t)}{L(t)} d t \exp \int_{0}^{|\arg z|} \frac{L^{\prime}\left(|z| e^{i \theta}\right)}{L\left(|z| e^{i \theta}\right)}|z| d \theta \\
& \leqslant L(N) \exp \left[(2 \epsilon /(2+\alpha \pi))\left(|z|^{\alpha}-N^{\alpha}\right)\right] \exp \left[(\alpha \pi \epsilon /(2+\alpha \pi))|z|^{\alpha}\right] \\
& \leqslant L(N) \exp \left[-2 \epsilon N^{\alpha} /(2+\alpha \pi)\right] e^{\epsilon|z|^{\alpha}} .
\end{aligned}
$$

The lemma follows.

The next lemma provides the connection between functions regularly varying at infinity of order $\alpha>0$ and functions of regular type $\alpha>0$.

LEMMA 9. If $C(z)=\exp (-G(-i z))$, then $G(z)$ is of regular type $\alpha>0$ iff $C(z)=e^{-\beta(-i z)^{\alpha}} L(-i z)$, where $\beta>0$ and $L(z)$ is regularly varying at infinity of order $\alpha$ in some half plane $x \geqslant \eta$.

The proof of this is similar to the proof of Lemma 7. In view of this result, any function of the form $G(z)=z^{\alpha}+\log L(z)$, with $L(z)$ regularly varying at infinity of order $\alpha$ in some half plane $x \geqslant \eta$, lies in $G$.

- Now if $C(z)=e^{-\beta(-i z)^{\alpha}} L(z)$, where $\beta>0$ and $L(z)$ is regularly varying at infinity of order $\alpha$, we are in a position to apply Theorem 6 to the function $C(z)$. The next result provides the necessary control on the argument of $C(z)$. 
LEMMA 10. Let $C(z)=e^{-\beta(-i z)^{\alpha}} L(-i z)$, where $\beta>0$ and $L(z)$ is regularly varying at infinity of order $\alpha$ in some half plane $x \geqslant \eta$. Then $h^{-}(\theta ; C)=h^{+}(\theta ; C)$ $=+\infty$ for $0<\theta \leqslant \pi / 2$.

The proof of this is similar to the proof of Theorem 7, and is left to the reader.

As we observed above, if $0<\alpha<1$ and $G(z)=z^{\alpha}+\log L(z)$, where $L(z)$ is regularly varying at infinity of order $\alpha$ in some half plane $x \geqslant \eta$, then $G \in G$. The next result provides us with a simple asymptotic structure $\Phi$ such that $G \in$ $G(\Phi)$.

THEOREM 10. Let $L(z)$ be regularly varying at infinity of order $\alpha$ in a half plane $x \geqslant \eta$, and let $G(z)=z^{\alpha}+\log L(z)$. Then $G \in G(\Phi)$, where $\Phi=$ $\{\phi(z)=z\}$.

Proof. By Lemma 8 , for any $\epsilon>0$ there is a constant $C(\epsilon)>0$ such that

$$
C(\epsilon)^{-1} e^{-\epsilon|z|^{\alpha}} \leqslant|L(z)| \leqslant C(\epsilon) e^{\epsilon|z|^{\alpha}},
$$

for all $z$ in the half plane $x \geqslant \eta$. Let $\delta>0$ be chosen so that $\cos \alpha \theta \geqslant \delta$ for $0 \leqslant \theta \leqslant \pi / 2$, and let $\epsilon$ be chosen so that $0<\epsilon<\delta$. Let $z_{1}$ and $z_{2}$ be points in the half plane $x \geqslant \eta$ such that $\left|\operatorname{Re} G\left(z_{1}\right)-\operatorname{Re} G\left(z_{2}\right)\right| \leqslant \epsilon$. Then

$$
e^{-\epsilon} \leqslant\left|L\left(z_{2}\right) e^{z_{2}^{\alpha}} / L\left(z_{1}\right) e^{z_{1}^{\alpha}}\right| \leqslant e^{\epsilon},
$$

and thus we obtain

$$
\begin{aligned}
& C(\epsilon)^{-1} e^{-(1+\epsilon)\left|z_{1}\right|^{\alpha}} \leqslant\left|L\left(z_{1}\right)\right|^{-1} e^{-\left(\cos \left(\alpha \arg z_{1}\right)\right)\left|z_{1}\right|^{\alpha}}=\mid L\left(z_{1}\right)^{-1} e^{-z_{1}^{\alpha} \mid}
\end{aligned}
$$

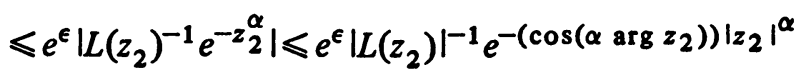

$$
\begin{aligned}
& \leqslant C(\epsilon) e^{\epsilon} e^{-(\delta-\epsilon)\left|z_{2}\right|^{\alpha}} \text {. }
\end{aligned}
$$

Taking logarithms, we obtain

$$
-\log C(\epsilon)-\epsilon+(\delta-\epsilon)\left|z_{2}\right|^{\alpha} \leqslant \log C(\epsilon)+(1+\epsilon)\left|z_{1}\right|^{\alpha},
$$

from which it follows that $\left|z_{2} / z_{1}\right| \leqslant K$ for some constant $K>0$. The result is now immediate.

Combining Theorem 10 and Lemma 9 with Theorem 3, we obtain at once the following result.

THEOREM 11. Let $C(z)=e^{-\beta(-i z)^{\alpha}} L(-i z)$, where $\beta>0,0<\alpha<1$, and $L(z)$ is regularly varying at infinity of order $\alpha$ in some half plane $x \geqslant \eta$. Suppose that $A \in \Pi^{\infty}$, and that $A$ can be written in the form

$$
A(z)=C(z)[1+P(z)] \text {. }
$$


where $P(z)=O\left(\left.\left.\right|_{z}\right|^{\alpha-1}\right)$ as $z \rightarrow \infty$ in some closed half plane $y \geqslant \eta^{\prime}$. Then $T\left(A+e^{i z} \Pi^{\infty}\right)$ and $T\left(C+e^{i z} \Pi^{\infty}\right)$ are similar.

8. Examples. We conclude this paper with a number of concrete examples to illustrate the foregoing material. We begin with some examples of operators having symbols of regular type.

(a) Regular type 0. Numerous examples of this case were given in [9]. Here we only summarize these examples, and present some additional ones.

It was observed in [9] that this case contains the classical fractional integration operators defined for $\alpha>0$ by

$$
J^{\alpha}: f(x) \rightarrow \Gamma(\alpha)^{-1} \int_{0}^{x}(x-t)^{\alpha-1} f(t) d t .
$$

(Symbol $C(z)=1 /(-i z)^{\alpha}$.) It was shown in particular that the earlier result of Kalisch [15] and Sahnovič [22] concerning operators similar to integral powers of $J$ can easily be deduced from Theorem 9 . Theorem 9 was also used to derive an analogue of the Kalisch-Sahnovič result for nonintegral powers of $J$. (See [9, Theorem 10].)

We mention some additional operators having symbols of the form $L(-i z) /(-i z)^{\alpha}$, where $\alpha>0$ and $L(z)$ is slowly varying at infinity. The classical logarithmic kernel operators of Volterra and Pérès [29], defined for $\alpha>0$ by

$$
L_{\alpha}: f(x) \rightarrow \Gamma(\alpha)^{-1} \int_{0}^{x} t^{\alpha-1}\left[-\log t+\Gamma^{\prime}(\alpha) / \Gamma(\alpha)\right] f(x-t) d t,
$$

have symbols $C(z)=\log (-i z) /(-i z)^{\alpha}$. The operators $M_{\alpha}$ defined for $\alpha>0$ by

$$
M_{\alpha}: f(x) \rightarrow \int_{0}^{x} \nu(t, \alpha-1) f(x-t) d t,
$$

where $\nu(t, \alpha-1)=\int_{\alpha}^{\infty} t^{s-1} d s / \Gamma(s)$ (see Erdélyi et al. [7]), have symbols $C(z)=$ $1 /(-i z)^{\alpha} \log (-i z)$. Both of these examples were discussed in [9]. Another simple example (not given in [9]) is afforded by

$$
T_{\alpha}: f(x) \rightarrow \int_{0}^{x} t^{(\alpha-1) / 2} J_{\alpha-1}\left(2 t^{1 / 2}\right) f(x-t) d t,
$$

where $\alpha>0$ and $J_{\nu}(z)$ is a Bessel function (see Watson [30]). Note that $T_{\alpha}$ has symbol $C(z)=e^{-i / z} /(-i z)^{\alpha}$. Using Theorem 9 , one can easily show that $T_{\alpha}$ is similar to $J^{\alpha}$.

As an additional illustration of Theorem 9, we next consider a more complicated example. For $\alpha>0$, let

$$
R_{\alpha}: f(x) \rightarrow \Gamma(\alpha)(2 \pi)^{-1 / 2} \int_{0}^{x} e^{-t^{2} / 4}\left[D_{-2 \alpha}(-t)-D_{2 \alpha}(t)\right] f(x-t) d t
$$

where $D_{\nu}(z)$ is a parabolic cylinder function (see Erdélyi et al. [7]). We show that $R_{\alpha}$ is similar to $J^{\alpha+1}$. To see this, note that $R_{\alpha}$ has symbol 
$C(z)=(-i z)^{-2 \alpha} e^{(-i z)^{2} / 2} \Gamma\left(\alpha, 1 / 2(-i z)^{2}\right)$, where $\Gamma(\alpha, z)$ is an incomplete gamma function (see Erdélyi et al. [7]). Then using the asymptotic expansion for the incomplete gamma function (see [1]), we have

$$
C(z) \approx(-i z)^{-\alpha-1}[1+(\alpha-1) /(-i z)+\cdots]
$$

as $z \rightarrow \infty$ in any upper half plane $y \geqslant \delta, \delta>0$. The assertion then follows from Theorem 9 .

(b) Regular type $\alpha>0$. An interesting class of operators having symbols of this type are the operators $N_{\rho}$ defined for any real number $\rho$ by

$$
N_{\rho}: f(x) \rightarrow \pi^{-1 / 2} \int_{0}^{x} t^{\rho} e^{-1 / t} f(x-t) d t .
$$

In particular, it was shown in [9] that if $\rho=-3 / 2, N_{\rho}$ is nonunicellular. See also [11]. It is not difficult to show that $N_{\rho}$ has symbol $C_{\rho}(z)=$ $2 \pi^{-1 / 2}(-i z)^{-(\rho+1) / 2} K_{\rho+1}\left(2(-i z)^{1 / 2}\right)$, where $K_{\nu}(z)$ is a modified Bessel function (see Watson [30]). We assert that $C_{\rho}(z)$ is of the form $C_{\rho}(z)=\exp (-G(-i z)$ ), where $G$ is a function of regular type $1 / 2$. To see that this is so, we utilize the asymptotic expansions of $K_{\nu}(z)$ and $K_{\nu}^{\prime}(z)$ (see [1]). We have

$$
\begin{aligned}
-C_{\rho}^{\prime}(i z) / C_{\rho}(i z) & =1 / 2(\rho+1) z^{-1}+z^{-1 / 2} K_{\rho+1}^{\prime}\left(2 z^{1 / 2}\right) / K_{\rho+1}\left(2 z^{1 / 2}\right) \\
& =z^{-1 / 2}\left[1 / 2(\rho+1) z^{-1 / 2}+\left(1+F_{1}(z)\right) /\left(1+F_{2}(z)\right)\right] \\
& =z^{-1 / 2}\left\{1+1 / 2(\rho+1) z^{-1 / 2}+\left[\left(1+F_{1}(z)\right) /\left(1+F_{2}(z)\right)-1\right]\right\},
\end{aligned}
$$

where $F_{1}(z), F_{2}(z)=O\left(|z|^{-1 / 2}\right)$ as $z \rightarrow \infty$. Thus we see that $-C_{\rho}^{\prime}(i z) / C_{\rho}(i z)$ $=z^{-1 / 2}[1+o(1)]$ as $z \rightarrow \infty$ in the half plane $x \geqslant \delta$, for any $\delta>0$. The assertion follows.

Now from the expansion

$$
C_{\rho}(z)=(-i z)^{-1 / 2(\rho+3 / 2)} e^{-2(-i z)^{1 / 2}}\left[1+O\left(|z|^{-1 / 2}\right)\right]
$$

as $z \rightarrow \infty$, and from Theorem 11 , it follows that $T\left(C_{\rho}+e^{i z} \Pi^{\infty}\right)$ is similar to $T\left(\widetilde{C}_{\rho}+e^{i z} \Pi^{\infty}\right)$, where $\widetilde{C}_{\rho}(z)$ is given by

$$
C_{\rho}(z)=(-i z)^{-1 / 2(\rho+3 / 2)} e^{-2(-i z)^{1 / 2}} .
$$

It follows that for $\rho>-3 / 2, N_{\rho}$ is similar to $J^{(p+3 / 2) / 2} N_{-3 / 2}$, and for $\rho<$ $-3 / 2$. $N_{-3 / 2}$ is similar to $J^{-(\rho+3 / 3) / 2} N_{\rho}$.

As a further illustration, let $\nu$ be any real number, and consider the operators

$$
S_{\nu}: f(x) \rightarrow(2 \pi)^{-1 / 2} \int_{0}^{x} t^{-1} e^{-1 / 2 t} W_{1 / 2, \nu}(1 / t) f(x-t) d t
$$


where $W_{\mu, \nu}(z)$ is a Whittaker function (see Erdélyi et al. [7]). We assert that $S_{\nu}$ is similar to $N_{-3 / 2}$ for all $\nu$. To see this, note that $S_{\nu}$ has symbol

$$
A_{\nu}(z)=(2 / \pi)(-i z)^{1 / 2} K_{\nu+1 / 2}\left((-i z)^{1 / 2}\right) K_{\nu-1 / 2}\left((-i z)^{1 / 2}\right) .
$$

Again utilizing the asymptotic expansion for $K_{\nu}(z)$, we obtain

$$
\begin{aligned}
A_{\nu}(z) & =(2 / \pi)(-i z)^{1 / 2}\left(\pi / 2(-i z)^{1 / 2}\right) e^{-2(-i z)^{1 / 2}}\left[1+O\left(1 /|z|^{1 / 2}\right)\right] \\
& =e^{-2(-i z)^{1 / 2}\left[1+O\left(1 /|z|^{1 / 2}\right)\right]}
\end{aligned}
$$

as $z \rightarrow \infty$. The assertion then follows from Theorem 11. In particular, it follows that $S_{\nu}$ is nonunicellular for all $\nu$.

(c) A more delicate example. Throughout this paper we have obtained various results on similarity by utilizing a special case of the similarity criterion of $\S 1$; namely, we have consistently restricted our attention to cases in which the lifting $\theta(z)$ satisfies the condition $\theta(z)-z \in \Pi^{\infty}$, rather than attempting to utilize the criterion in its fullest generality. As an illustration of how this extra generality can be used, let us consider the operator $J\left(I+i L_{1}\right)$, where $L_{1}$ is defined by (35) with $\alpha=1$. We assert that $J\left(I+i L_{1}\right)$ is similar to $J$. The reader can easily convince himself that this can in no way be deduced from Theorem 6 or any of its corollaries. We shall apply the similarity criterion of $\$ 1$ directly, and in its full generality. First note that $J\left(I+i L_{1}\right)$ has symbol

$$
\begin{aligned}
A(z) & =(-i z)^{-1}[1-\log (-i z) / z]=(1 /(-i))\left(1 / z^{2}\right)[z-\log (-i z)] \\
& =1 /\left[-i z^{2} /(z-\log (-i z))\right] \\
& =1 /-i[z+z \log (-i z) /(z-\log (-i z))]=1 /(-i \theta(z)),
\end{aligned}
$$

where $\theta(z)=z+z \log (-i z) /(z-\log (-i z)) \equiv z+\chi(z)$. To prove the desired similarity, we must show that $\chi(z)$ satisfies the hypotheses of Theorem 3 . It is a routine exercise to show that $\operatorname{Im} \chi(z)$ is bounded. One also easily checks that $\operatorname{Re} \chi(z)$ is not bounded. Clearly $\chi(z)=o(|z|)$ as $z \rightarrow \infty$. Finally, note that

$$
\chi^{\prime}(z)=\left(z-(\log (-i z))^{2}\right) /(z-\log (-i z))^{2} \rightarrow 0
$$

as $z \rightarrow \infty$. It follows that $J$ and $J\left(I+i L_{1}\right)$ are similar, as stated.

The last example provides an excellent illustration of the extremely delicate nature of the similarity problem for convolution operators. The results we have obtained above tend, in a small way, to give support to the idea that similarity classes of operators of the form (1) should somehow be identifiable in terms of their symbols. However, the last example serves to make one painfully aware of just how delicate such an identification process would have to be, particularly when viewed in comparison with the work of Kalmuševskii [18]. Specifically, 
Kalmuševskii's results imply that $J\left(I+L_{1}\right)$ is not similar to $J$. It is difficult to assess the true significance of this phenomenon in the light of what is presently known, or even to formulate a reasonable conjecture as to what might lie behind it. However, the following comments are perhaps of some value.

Let $T$ be an operator of the form (1), and assume that $T$ has a symbol $A(z)=(-i z)^{-1}[1+P(z)]$. Under what circumstances will $T$ be similar to $J$ ? According to Theorem 9, if $P(z)=O(1 /|z|)$ as $z \rightarrow \infty$, then $T$ will be similar to $J$. No additional information on $P(z)$ is needed. Merely knowing that $P(z)$ has the given order of growth is enough to insure similarity. However, as the above example illustrates, this order of growth is not necessary for similarity. It is still possible for $T$ to be similar to $J$ with slightly looser control on $P(z)$, namely $P(z)=$ $O(\log z|/| z \mid)$ as $z \rightarrow \infty$. However, as the Kalmuševskii result shows, when the control on $P(z)$ is relaxed in this way, it is no longer sufficient to know merely that $P(z)$ has this order of growth. It is necessary to compensate for the reduction in the order of the growth of $P(z)$ by having additional information about the function. Exactly what shape this additional information may take awaits further research.

\section{REFERENCES}

1. M. Abramowitz and I. A. Stegun (Editors), Handbook of mathematical functions with formulas, graphs and mathematical tables, National Bureau of Standards Appl. Math. Series, 55, Superintendent of Documents, U. S. Government Printing Office, Washington, D. C., 1964. MR 29 \#4914.

2. L. V. Ahlfors and L. Sario, Riemann surfaces, Princeton Math. Ser., no. 26, Princeton Univ. Press, Princeton, N. J., 1960. MR 22 \#5729.

3. M. S. Brodskiî, Triangular and Jordan representations of linear operators, "Nauka", Moscow, 1969; English transl., Transl. Math. Monographs, vol. 32, Amer. Math. Soc., Providence, R. I., 1971. MR 41 \#4283.

4. V. W. Daniel, Invariant subspaces of convolution operators, University of Virginia Doctoral Dissertation, 1970.

5. Convolution operators on Lebesgue spaces of the half line, Trans. Amer. Math. Soc. 164 (1972), 479-488. MR 45 \#939.

6. P. L. Duren, Theory of $H^{p}$ spaces, Pure and Appl. Math., vol. 38, Academic Press, New York, 1970. MR 42 \#3552.

7. A. Erdélyi et al., Tables of integral transforms. Vol. I, McGraw-Hill, New York, 1954. MR 15, 868 .

8. W. Feller, An introduction to probability theory and its applications. Vol. II, Wiley, New York, 1966. MR 35 \#1048.

9. R. E. Frankfurt and J. L. Rovnyak, Finite convolution operators, J. Math Anal. Appl. 49 (1975), 347-374.

10. J. M. Freeman, Volterra operators similar to $J: f(x) \rightarrow \int_{0}^{x} f(y) d y$, Trans. Amer. Math. Soc. 116 (1965), 181-192. MR 33 \#592.

11. J. I. Ginsberg and D. J. Newman, Generators of certain radical algebras, J. Approximation Theory 3 (1970), 229-235. MR 41 \#9014.

12. I. C. Gohberg and M. G. Krein, Introduction to the theory of linear nonselfadjoint operators in Hilbert space, "Nauka", Moscow, 1965; English transl., Transl. Math. Monographs, vol. 18, Amer. Math. Soc., Providence, R. I., 1969. MR 36 \#3137; 39 \#7447. 
13. I. C. Gohberg and M. G. Kreĭn, Theory and applications of Volterra operators in Hilbert space, "Nauka", Moscow, 1967; English transl., Transl. Math. Monographs, vol. 24, Amer. Math. Soc., Providence, R. I., 1970. MR 36 \#2007; 41 \#9041.

14. G. H. Hardy, Orders of infinity, Cambridge Univ. Press, London, 1924.

15. G. K. Kalisch, On similarity, reducing manifolds, and unitary equivalence of certain Volterra operators, Ann. of Math. (2) 66 (1957), 481-494. MR 19, 970.

16. On isometric equivalence of certain Volterra operators, Proc. Amer. Math. Soc. 12 (1961), 93-98. MR 22 \#11260.

17. On similarity invariants of certain operators in $L_{p}$, Pacific J. Math. 11 (1961), 247-252. MR 22 \#11261.

18. I. I. Kalmuševskir, On the linear equivalence of Volterra operators, Uspehi Mat. Nauk 20 (1965), no. 6 (126), 93-97. (Russian) MR 32 \#8161.

19. B. Ja. Levin, Distribution of zeros of entire functions, GITTL, Moscow, 1956; English transl., Transl. Math. Monographs, vol. 5, Amer. Math. Soc., Providence, R. I., 1964. MR 19, 402; 28 \#217.

20. S. J. Osher, Two papers on similarity of certain Volterra integral operators, Mem. Amer. Math. Soc. No. 73 (1967). MR 36 \#2018.

21. L. A. Sahnovič, On the reduction of Volterra operators to the simplest form and on inverse problems, Izv. Akad. Nauk SSSR Ser. Mat. 21 (1957), 235-262. (Russian) MR $19,970$.

22. Spectral analysis of operators of the form $K f=\int_{0}^{x} f(t) k(x-t) d t$, Izv.

Akad. Nauk SSSR Ser. Mat. 22 (1958), 299-308. (Russian) MR 20 \#5409.

23. - Spectral analysis of Volterra operators defined on the vector-function space $L_{m}^{2}(0, l)$, Ukrain. Mat. Ž. 16 (1964), 259-268; English transl., Amer. Math. Soc. Transl. (2) 61 (1967), 85-95. MR 29 \#2680.

24. D. Sarason, A remark on the Volterra operator, J. Math. Anal. Appl. 12 (1965), 244-246. MR 33 \#580.

25. - Generalized interpolation in $H^{\infty}$, Trans. Amer. Math. Soc. 127 (1967), 179-203. MR $34 \$ 8193$.

26. E. H. Spanier, Algebraic topology, McGraw-Hill, New York 1966. MR 35 \#1007.

27. B. Sz.-Nagy and C. Foias, Analyse harmonique des opérateurs de l'espace de Hilbert, Masson, Paris; Akad. Kiadó, Budapest, 1967; English rev. transl., North-Holland, Amsterdam; American Elsevier, New York; Akad. Kiadó, Budapest, 1970. MR 37 \#778; 43 \#947.

28. V. Volterra, Theory of functionals and of integral and integro-differential equations, Dover, New York, 1959. MR 20 \#7193.

29. V. Volterra and J. Pérès, Leçons sur la composition et les fonctions permutables, Gauthier-Villars, Paris, 1924.

30. G. N. Watson, $A$ treatise on the theory of Bessel functions, 2 nd ed., Cambridge Univ. Press, Cambridge; Macmillan, New York, 1944. MR 6, 64.

\section{SCHOOL OF MATHEMATICS, UNIVERSITY OF MINNESOTA, MINNEAPOLIS, MINNESOTA 55455}

Current address: Department of Mathematics, University of Kentucky, Lexington, Kentucky 40506 